\title{
Self-organization of Ce adatoms on Ag(111): A kinetic Monte Carlo study
}

\author{
N. N. Negulyaev, ${ }^{1}$ V. S. Stepanyuk, ${ }^{2}$ L. Niebergall, ${ }^{2}$ W. Hergert, ${ }^{1}$ H. Fangohr, ${ }^{3}$ and P. Bruno ${ }^{2}$ \\ ${ }^{1}$ Fachbereich Physik, Martin-Luther-Universität, Von-Seckendorff-Platz, 1, D-06120 Halle, Germany \\ ${ }^{2}$ Max-Planck-Institut für Mikrostrukturphysik, Weinberg 2, D-06120 Halle, Germany \\ ${ }^{3}$ School of Engineering Sciences, University of Southampton, Southampton SO17 1BJ, United Kingdom
}

(Received 31 January 2006; revised manuscript received 9 May 2006; published 18 July 2006)

One of the most fascinating experimental results in fabrication of artificial nanostructures is the creation of the macroscopically ordered superlattice of Ce adatoms on $\mathrm{Ag}(111)$ [F. Silly et al., Phys. Rev. Lett. 92, 016101 (2004)]. Here, performing kinetic Monte Carlo simulations, we study the formation of Ce superlattice at the atomic scale. It is demonstrated that the surface-state mediated long-range interaction between Ce adatoms can lead to their self-assembly into a well ordered structure. The temperature of the substrate and the concentration of Ce adatoms are shown to play a key role in this process.

DOI: 10.1103/PhysRevB.74.035421

PACS number(s): 68.35.Fx, 81.10.Aj, 68.37.Ef, 81.16.Dn

\section{INTRODUCTION}

One of the most important aspects in nanoscience is the formation of artificial nanostructures. Ordered structures of atoms and molecules can be generated either by the manipulation of single atoms or molecules ${ }^{1,2}$ by means of the tip of a scanning tunneling microscope (STM) or by using selfassembly processes of particles. ${ }^{3-6}$ Recent experimental and theoretical studies have indicated that the long-range interactions (LRIs) between adsorbates on metal surfaces ${ }^{7-9}$ can be exploited to create a new nanostructures. ${ }^{10,11}$ Such interactions are caused by the quantum interference of surface-state electrons scattered off adsorbates. Experiments performed by means of the STM (Refs. 4 and 10) and $a b$ initio calculations ${ }^{11,12}$ have resolved surface-state mediated interactions at large adatom-adatom separations. The LRI potential depends on the type of the adsorbate atoms and the substrate. The LRI decays as $1 / r^{2}$, where $r$ is the interatomic distance. It oscillates with a period of $\lambda_{F} / 2$. Schematic description of the LRI between adsorbates is depicted in Fig. 1.

It is the special form of the LRI potential which may crucially influence the arrangement of atoms at low temperatures and low coverage. Knorr et al. ${ }^{10}$ have proposed that if the thermal energy of adatoms is not sufficient to overcome the repulsive barrier $D$ (Fig. 1), the dimers are not formed, and a new ordered structures with the first nearest-neighbor (NN) adatom separation corresponding to the first minimum of the pair potential (Fig. 1) may be formed. Early attempts to exploit the LRI to create ordered superlattice failed. ${ }^{10}$ The breakthrough came with experiments of Silly et al. on Ce adatoms on $\operatorname{Ag}(111) .{ }^{13,14}$ It has been discovered that the $\mathrm{Ce}$ atoms form well ordered hexagonal superlattice with a lattice constant of $32 \AA$. The Ce superlattice is most nicely ordered at $3.9 \mathrm{~K}$. The LRI between Ce adatoms has been proposed to be the driving force for the self-organization of Ce adatoms. These experiments have indicated that sample temperature, low adatom diffusion barrier and adatom concentration are the key parameters for successful self-organization of $\mathrm{Ce}$ adatoms into the superlattice.

Here, performing kinetic Monte Carlo (KMC) simulations, we study the formation of the $\mathrm{Ce}$ superlattice on $\mathrm{Ag}(111)$ at the atomic scale. Our results give clear evidence that the LRI between Ce adatoms can lead to the formation of well ordered superlattice at low temperature. The stability of the superlattice is investigated.

\section{METHOD OF CALCULATION}

For our simulations we use the rejection-free KMC method of Fichthorn and Weinberg. ${ }^{15,16}$ The hop rate of an adatom from site $k$ to site $j$ on the (111) surface is calculated using the expression $v_{k \rightarrow j}=v_{0} \exp \left(-E_{k \rightarrow j} / k_{B} T\right)$, where $T$ is the temperature of the substrate, $v_{0}$ is the attempt frequency and $k_{B}$ is the Boltzmann factor. We set $v_{0}$ to $10^{12} \mathrm{~s}^{-1}$ for the Ag surface. ${ }^{13,14}$ The influence of the LRI on adatom diffusion is included in the hopping barrier which takes the form ${ }^{16}$ $E_{k \rightarrow j}=E_{D}+0.5\left(E_{j}-E_{k}\right)$. Here $E_{D}$ is the diffusion barrier for an isolated atom on a clean surface. ${ }^{14} E_{k(j)}$ is the sum of pair potentials due to the LRI of the considered adatom with all the other adatoms, if the adatom is at site $k(j)$. Therefore $0.5 *\left(E_{j}-E_{k}\right)$ describes an additional barrier for dimer formation.

For the further discussions we transform the expression $v_{k \rightarrow j}$ to the following form:

$$
v_{k \rightarrow j}=v_{D} \exp \left[-0.5\left(E_{j}-E_{k}\right) / k_{B} T\right],
$$

where $v_{D}=v_{0} \exp \left(-E_{D} / k_{B} T\right)$ is the average hop rate of the isolated adatom on the clean surface.

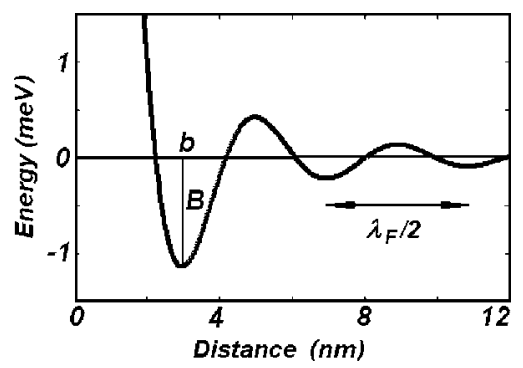

FIG. 1. LRI potential between Ce adatoms on $\mathrm{Ag}(111)$ calculated using Eq. (2) with a values of parameters given in Sec. II. The repulsive part of the potential is on the distances $0.5-2.0 \mathrm{~nm}$, its magnitude $D$ is $13.8 \mathrm{meV}$. Other parameters are: $B=-1.1 \mathrm{meV}, b$ $=2.96 \mathrm{~nm}$. 
In principle, one has to distinguish between fcc and hcp sites on a fcc (111) surface. A difference in binding energy for $\mathrm{Ce}$ at these two sites cannot be seen in the experiment. ${ }^{13,14}$ Therefore, fcc and hcp sites are considered in our simulations to be identical.

The interaction between two $\mathrm{Ce}$ adatoms on $\mathrm{Ag}(111)$ surface is calculated using the model of Hyldgaard and Persson $^{17}$

$$
E(r)=A_{0} E_{0}\left(\frac{2 \sin \delta_{0}}{\pi}\right)^{2} \frac{\sin \left(2 k_{F} r+2 \delta_{0}\right)}{\left(k_{F} r\right)^{2}},
$$

where the parameters are taken from the experimental work. ${ }^{14}$ Here $k_{F}$ is the Fermi surface wave vector $\left(0.813 \mathrm{~nm}^{-1}\right), E_{0}$ is the surface state band edge relative to the Fermi energy $(-63.0 \mathrm{meV})$. The other parameters are the phase shift $\delta_{0}=(0.37 \pm 0.05) \pi$ and the scattering amplitude $A_{0}=0.27 \pm 0.03$. We perform simulations with the following values of the parameters $A_{0}=0.30, \delta_{0}=0.42 \pi$. These parameters lead to the potential given in Fig. 1. Repulsive part of the potential, which includes local maximum $D$, prevents dimers formation. In this work we concentrate on the local minimum $B$, responsible for the formation of LRI stabilized nanostructures, particularly, superlattice.

Equation (2) has been obtained through an asymptotic evaluation of the one-electron contributions to the interaction energy. However, even for large adatom-adatom separations the completely asymptotic behavior may not be fully reached. There have been important studies on indirect interactions between atoms which have investigated a preasymptotic behavior of the interaction energy. ${ }^{18,19}$ For example, Gumhalter and Brenig ${ }^{19}$ have shown that indirect interactions between adsorbates in quasi-one-dimensional (Q1D) gas is determined by the two terms, the one which decays exponentially with the distance from the impurity, and the other which oscillates. The term falling off exponentially is responsible for the effects brought about by the intraorbital screening charge whereas the oscillatory term describes the extra-atomic effects mediated by the Q1D electron gas. It has been found that there is a smooth transition between these two regimes of screening.

Theoretical studies ${ }^{17}$ and our own ab initio calculations ${ }^{12}$ have shown that in the case of $\mathrm{Cu}(111)$, the asymptotic equation is accurate even down to a distance of $\lambda_{F} / 2 \approx 15 \AA\left(\lambda_{F}\right.$ is a surface state Fermi wavelength). The $\lambda_{F}$ of $\operatorname{Ag}(111)$ is more than twice larger than that of $\mathrm{Cu}(111)$. This means that the product $k_{F} r\left(k_{F}\right.$ is the surface-state Fermi vector) is relatively small and asymptotic regime may not be reached even for large separations $r$. Experiments of Knorr et al ${ }^{10}$ have shown that the pair interaction energy between Co adatoms on $\operatorname{Ag}(111)$ exhibits an oscillatory behavior with the period of $\lambda_{F} / 2 \approx 38 \AA$ clearly seen already at $27-28 \AA$. The studies of an isolated $\mathrm{Ce}$ adatom on $\operatorname{Ag}(111)^{14}$ have revealed the standing-wave patterns with the periodicity at $E_{F}$ given by $\lambda_{F} / 2 \approx 38 \AA$ for distances larger than $\approx 25 \AA$. Thus, presumably the asymptotic regime on $\operatorname{Ag}(111)$ is already reached for $r>20-25 \AA$.

To demonstrate that for such adatom-adatom separations Eq. (2) correctly describes the magnitude of the interaction energy, we have performed a fully $a b$ initio calculations of the depth of the potential well (responsible for the formation of the superlattice) corresponding to the first minimum of the pair potential (Fig. 1). From the experimental side,${ }^{14}$ the pair potential has been determined by making a statistical analysis of the motion of Ce adatoms in the superlattice confining potential. The pair potential well depth has been found to be about $0.8 \mathrm{meV}$. Our ab initio calculations are based on density functional theory and multiple-scattering approach using the Korringa-Kohn-Rostoker Green's function method for low-dimensional systems. ${ }^{12,20}$ The main contribution to the interaction energy at large adatom-adatom separations is well approximated by the single-particle energies alone. ${ }^{12,21,22} \mathrm{We}$ perform calculations of the interaction energies in the framework of the frozen potential approximation and use the Lloyd's formula for calculations of the single-particle energies. ${ }^{21}$ As a benefit of this approach, calculations can be performed up to very large distances and very small interaction energies can be resolved. Several applications of our method can be found in recent works. ${ }^{11,22,23}$ We have found that the pair potential well depth is $0.6 \mathrm{meV}$ which is in reasonable agreement with experiments. Therefore, we believe that the asymptotic equation (2) fitted to the experimental results properly describes the substrate-mediated interaction between $\mathrm{Ce}$ adatoms.

In simulations LRI between more than two adatoms are described by pairwise summation. Our ab initio calculations and other studies have shown that such approximation is well justified. ${ }^{12,16}$ Periodic boundary conditions in the surface are applied in all calculations. The size of the simulation cell is $50 \mathrm{~nm} \times 50 \mathrm{~nm}$. A cutoff radius of $4.1 \mathrm{~nm}$ is used in the simulations.

For the analysis of results of KMC simulations we use an atom distribution function described below. Usually the radial distribution function (RDF) is calculated for homogeneous and isotropic systems to characterize structural correlations:

$$
f(r)=\frac{S}{4 \pi r N^{2}}\left\langle\sum_{i=1}^{N} \sum_{j \neq i} \delta\left(r-r_{i j}\right)\right\rangle,
$$

where $S$ is a square of the considered simulation cell. Function $f(r)$ gives the probability to find an adatom at a distance $r$ if an adatom at the origin of the coordinate system is considered.

To study the formation of the superlattice in dependence on temperature we will keep the angular information, i.e., we avoid the angular averaging of the pair distribution function. Therefore the angular-resolved atomic density function (ARADF), defined in Eq. (4) is used.

$$
g(\mathbf{r})=\frac{A}{N^{2}}\left\langle\sum_{i=1}^{N} \sum_{j \neq i} \delta\left[\mathbf{r}-\left(\mathbf{r}_{j}-\mathbf{r}_{i}\right)\right]\right\rangle .
$$

Here $A$ is a normalizing term, which can be chosen from the arbitrary condition, for example, from $\int_{D} g(\mathbf{r}) d S=1$, where $D$ is the whole definition area of a function $g(\mathbf{r})$. ARADF gives the probability to find an adatom at the position $\mathbf{r}$ if the second adatom is located at the origin of the coordinate sys- 

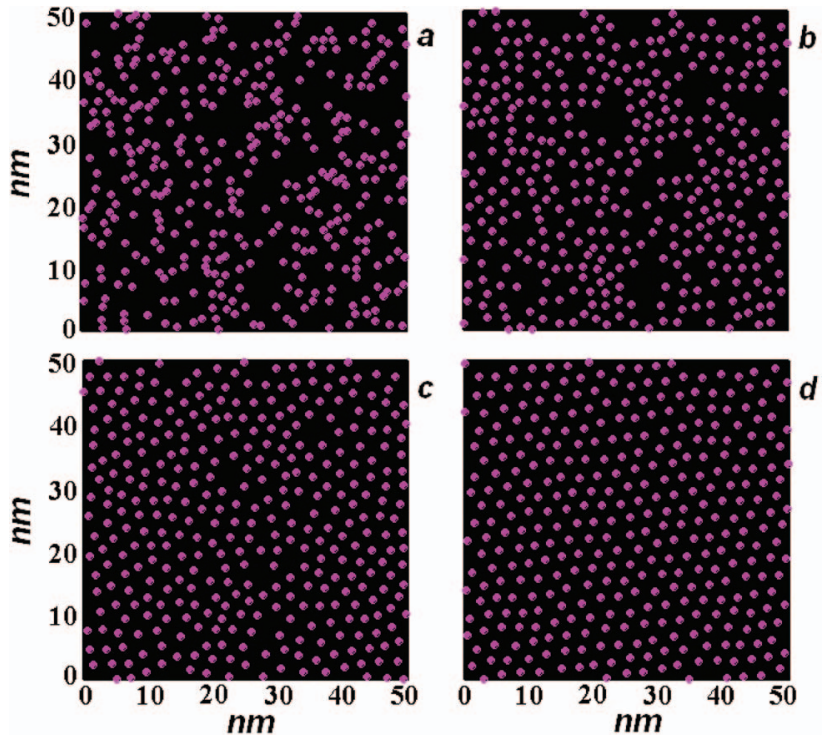

FIG. 2. (Color) The KMC simulation of $\mathrm{Ce}$ adatoms on $\mathrm{Ag}(111)$. The concentration is $1.0 \%$ of ML, temperature is $4.0 \mathrm{~K}$. These pictures correspond to the following number of KMC steps (a) 0 , (b) 700, (c) 7000, (d) 430000 or the time intervals (for a value of $E_{D}=9 \mathrm{meV}$ ) (a) $0 \mathrm{~s}$, (b) $0.4 \mathrm{~s}$, (c) $3.9 \mathrm{~s}$, (d) $240 \mathrm{~s}$. In (d) the distance between nearest $\mathrm{Ce}$ atoms is close to $3 \mathrm{~nm}$.

tem. Let us consider a system of $N$ atoms in its equilibrium state. To calculate $g(\mathbf{r})$ a square $S$ around each adatom with a side length $2 h$ is used to analyze the relative positions of the other adatoms. Let us take an arbitrary adatom in the system as the origin of the coordinate system. The value of $h$ has to be chosen to guarantee that at least the first neighbors in a hexagonal lattice are inside the square $S$. For the Ce superlattice at a coverage $1.0 \%$ of a ML the distance between

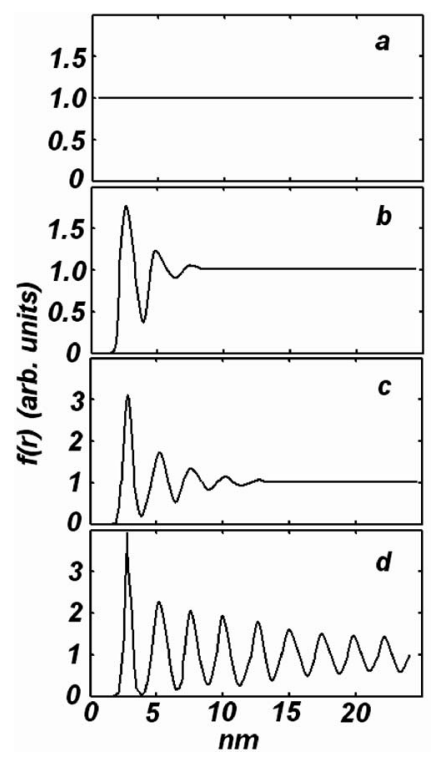

FIG. 3. The RDF for Ce adatoms on $\mathrm{Ag}(111)$ at a coverage of $1.0 \%$ of ML at a temperature of $4.0 \mathrm{~K}$. RDFs correspond to the following number of kMC steps: (a) 0, (b) 700, (c) 7000, (d) 430000 or the time intervals (for a value of $E_{D}=9 \mathrm{meV}$ ) (a) $0 \mathrm{~s}$, (b) $0.4 \mathrm{~s}$, (c) $3.9 \mathrm{~s}$, (d) $240 \mathrm{~s}$.

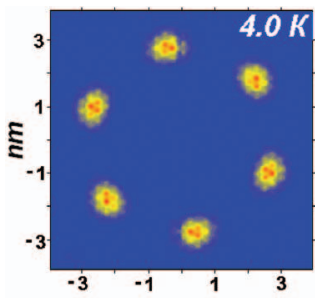

$g(r)$ (arb. units)
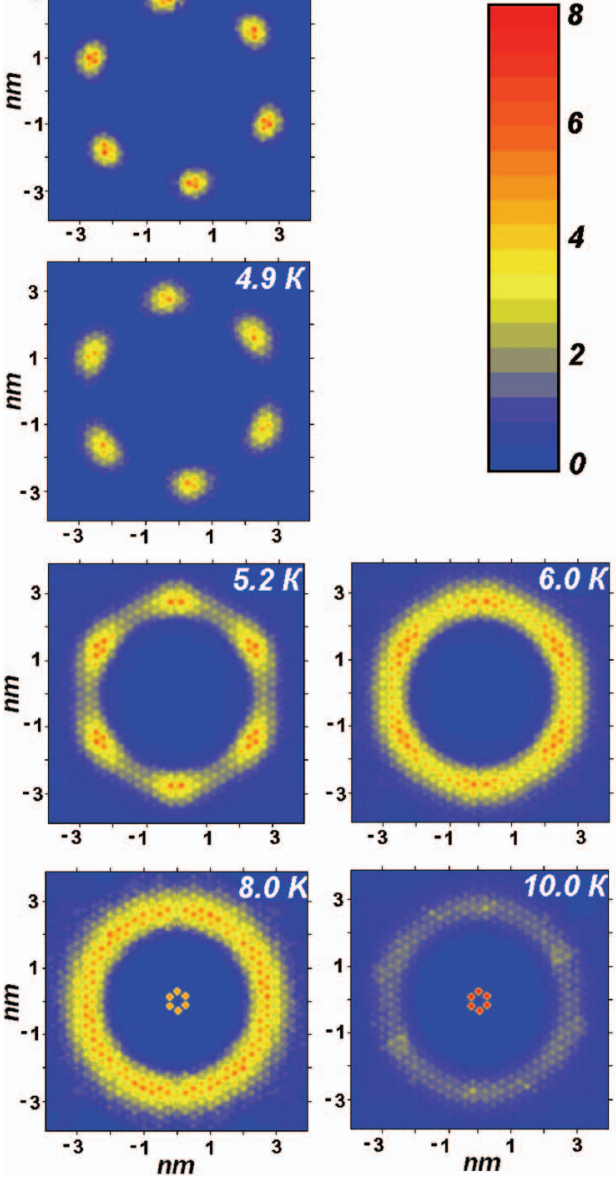

FIG. 4. (Color) Contour plots of the ARADF for Ce adatoms on $\mathrm{Ag}(111)$ at different temperatures; the concentration of Ce adatoms is $1.0 \%$ of ML.

neighboring particles is about $3.0 \mathrm{~nm}$. In this case the reasonable value for the magnitude of $h$ is about $4.0-5.0 \mathrm{~nm}$.

\section{RESULTS AND DISCUSSION}

Our simulations follow closely the experimental conditions of Silly et al. ${ }^{13,14}$ The two important questions have to

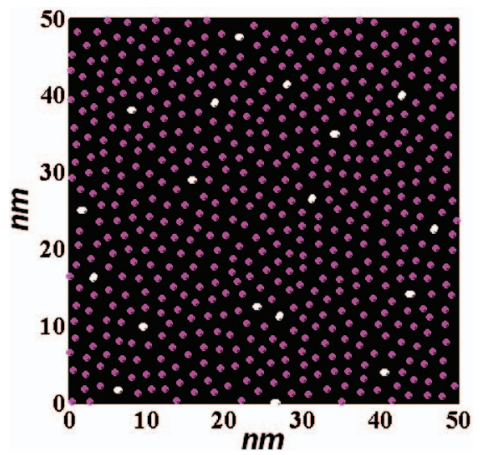

FIG. 5. (Color) The KMC simulation of $\mathrm{Ce}$ adatoms on $\mathrm{Ag}(111)$. The concentration is $1.8 \%$ of ML, temperature is $4.0 \mathrm{~K}$. White circles represent dimers which are formed immediately after the deposition. The distance between nearest $\mathrm{Ce}$ atoms is about $2.3 \mathrm{~nm}$. 
be answered: (i) at which coverage the superlattice is formed and (ii) is the superlattice stable? Simulations have been performed for the concentration range between $0.2-2.0 \%$. Below the concentration of $1.0 \%$ small hexagonal clusters and short chains are observed in good agreement with the experiment. ${ }^{24}$ Here we concentrate our discussion on the formation of the superlattice.

In Fig. 2 we present results of the KMC simulation for $\mathrm{Ce}$ on $\operatorname{Ag}(111)$ with a coverage of $1.0 \%$ at the temperature of $4.0 \mathrm{~K}$. These calculations nicely demonstrate the selforganization at the atomic scale. First, the randomly distributed atoms [Fig. 2(a)] self-assemble in small hexagonal clusters and form a short-range order [Fig. 2(b)]. Then, the hexagonal order is formed in regions with sizes of $10-15 \mathrm{~nm}$ [Fig. 2(c)], but long-range order is still absent. Finally, macroscopically ordered hexagonal two-dimensional superlattice occurs [Fig. 2(d)]. The distance between nearest Ce atoms is about $3.0 \mathrm{~nm}$ which corresponds to the position of the first minimum in the interaction potential for $\mathrm{Ce}$ on $\mathrm{Ag}(111)$ (Fig. 1). The RDF shown in Fig. 3 clearly demonstrates the formation of the macroscopic long-range order in Ce superlattice [Fig. 2(d)].

Figure 4 shows the ARADF for Ce adatoms on $\mathrm{Ag}(111)$ at a coverage $1.0 \%$ of a ML for the temperatures indicated in the figure. One can see the six separated peaks in the ARADF for 4.0 and $4.9 \mathrm{~K}$. The peaks for $4.0 \mathrm{~K}$ are slightly sharper than for $4.9 \mathrm{~K}$. Both plots correspond to the hexagonal order on the macroscopic scale. At $5.2 \mathrm{~K}$ the six peaks can be still seen, but they are blurred and start to form a ring. It means that long-range hexagonal order does not exist at this temperature and the $\mathrm{Ce}$ atoms move like particles in a "liquid state". There is, however, some local order: adatoms tend to have approximately $3 \mathrm{~nm}$ separation to each other. The long-range order will be destroyed if adatoms will have sufficient energy to leave the local minimum $B$ of the LRI potential (Fig. 1).

We have found that the melting temperature of Ce lattice on $\operatorname{Ag}(111)$ is about $5.0 \mathrm{~K}$. Figure 4 shows that at the temperature of $8.0 \mathrm{~K}$ the formation of dimers takes place (the six central peaks represent dimers). The distance between these peaks and the center of the square is equal to the nearest neighbor distance $a$ on $\operatorname{Ag}(111)$ surface $(0.289 \mathrm{~nm})$. At higher temperatures the density of dimers in the system increases. This is shown in the contour plot by a decreased intensity in the ring at $3 \mathrm{~nm}$ and an increased intensity in the six central peaks.

Depending on the temperature three different states of the superlattice are revealed by our simulations: the macroscopically ordered state, the "liquid state" characterized by chaotic motion of the adatoms, and the state characterized by the formation of dimers. The macroscopic ordered state exists when the temperature of the system is less than $5.0 \mathrm{~K}$, the "liquid state" is observed between 5.0 and $7.5 \mathrm{~K}$, dimer formation occurs for the temperature larger than $7.5 \mathrm{~K}$. The temperature interval in which the ordered state can be found is limited by some minimal temperature $T_{\text {cr }}$ that is determined by the average time $1 / v_{D}$ of a single adatom jump on the clean surface. The magnitude of $1 / v_{D}$ should have some reasonably large value, otherwise atoms do not hop and do

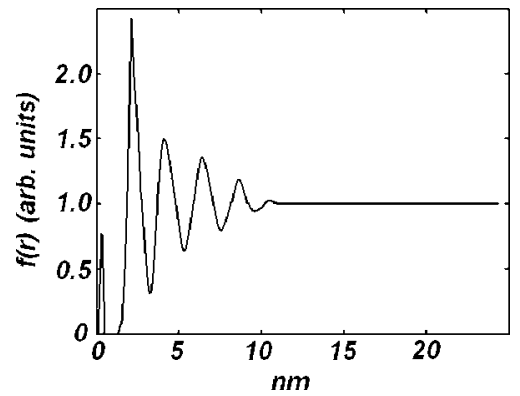

FIG. 6. The RDF for Ce adatoms on $\mathrm{Ag}(111)$ at coverage of $1.8 \%$ of ML; the temperature is $4.0 \mathrm{~K}$.

not self-assemble. If we assume that a reasonable time scale of one adatom hop is about $1 \mathrm{sec}$, we obtain for $T_{\text {cr }}$ a value of $3.9 \mathrm{~K}$. Therefore, there is a small temperature window (between $3.9 \mathrm{~K}$ and $5.0 \mathrm{~K}$ ) where $\mathrm{Ce}$ adatoms on $\mathrm{Ag}(111)$ can self-assemble into a superlattice. Note, that temperature windows for the ordered, "liquid" and the "dimer state" are determined only by the parameters of the LRI and do not depend on the magnitude of the diffusion barrier $E_{D}$. The prefactor $v_{D}$ in Eq. (1) which depends only on $E_{D}$ can be considered as the scale factor (constant) for all transition rates. Changing $E_{D}$ affects the characteristic time scale of the self-organization process but does not influence the relative transition probabilities.

Finally, we discuss the self-organization of $\mathrm{Ce}$ adatoms at coverage of $1.8 \%$ ML. Results of KMC simulations are shown in Fig. 5. There is a local hexagonal order in a many regions but a full macroscopic order is not observed. This is due to the fact that dimers are strongly pinned and their motion is found to be very unlikely. It should be noted that even a small number of dimers can drastically effect the possibility to create the superlattice. The RDF in Fig. 6 confirms the absence of a macroscopic long-range order, and reveals that a locally ordered structures exist only in rather small regions of $10-15 \mathrm{~nm}$.

Our calculations indicate that the maximal magnitude of the concentration which allows the emergence of a longrange ordered hexagonal structure is about $1.7 \%$ of a ML. At this coverage, the superlattice of $\mathrm{Ce}$ atoms is found to be compressed with the nearest neighbor distance of $2.3 \mathrm{~nm}$ whereas at $1.0 \%$ of a ML coverage (this is the "ideal" density for a hexagonal lattice) the nearest neighbor distance is equal to $b=2.89 \mathrm{~nm}$ (Fig. 1). These results are in good agreement with the experiment of Silly et al. ${ }^{14}$

\section{CONCLUSION}

We have presented the first KMC simulations of superlattices of adatoms stabilized by the substrate-mediated longrange interactions. Our results give a clear evidence that the LRI between adsorbates can be exploited to create new nanostructures. The formation of well ordered Ce superlattice on $\mathrm{Ag}(111)$ at the atomic scale is shown. Ab initio calculations have demonstrated that the interaction energy between two single Ce adatoms on $\operatorname{Ag}(111)$ is in agreement with experiments of Silly et al. ${ }^{13}$ Our studies have shown that the 
asymptotic form of the interaction energy properly describes the substrate-mediated interaction between Ce adatoms in the superlattice. We have established that self-organization of the superlattice $\mathrm{Ce} / \mathrm{Ag}(111)$ occurs when the concentration of adatoms is between 1.0 and $1.7 \%$ of $\mathrm{ML}$ and the temperature is $<5 \mathrm{~K}$. Increasing the temperature to $8 \mathrm{~K}$ leads to the formation of dimers and to the irreversible collapse of the superlattice. At concentrations $>1.7 \%$ of ML only a local hexagonal order is observed. It has been shown that a very delicate balance between the magnitude of diffusion barrier of single adatom, the depth of the first local minimum $B$ in LRI potential and the concentration of deposited adatoms must be implemented for the superlattice fabrication.

\section{ACKNOWLEDGMENTS}

This work was supported by Deutsche Forschungsgemeinschaft (DFG Grant Nos. SPP1165 and SPP1153).
${ }^{1}$ H. C. Manoharan, C. P. Lutz, and D. M. Eigler, Nature (London) 403, 512 (2000).

${ }^{2}$ A. J. Heinrich, C. P. Lutz, J. A. Gupta, and D. M. Eigler, Science 298, 1381 (2002).

${ }^{3}$ G. M. Whitesides and B. Grzybowski, Science 295, 2418 (2002).

${ }^{4}$ J. Repp, F. Moresco, G. Meyer, K.-H. Rieder, P. Hyldgaard, and M. Persson, Phys. Rev. Lett. 85, 2981 (2000).

${ }^{5}$ H. Brune, in Single Molecules at Surfaces, edited by F. Rosei, P. Grütter, and W. Hofer (Springer, New York, 2005).

${ }^{6}$ J. V. Barth, G. Costantini, and K. Kern, Nature (London) 437, 671 (2005).

${ }^{7}$ K. H. Lau and W. Kohn, Surf. Sci. 75, 69 (1978).

${ }^{8}$ A. Bogicevic, S. Ovesson, P. Hyldgaard, B. I. Lundqvist, H. Brune, and D. R. Jennison, Phys. Rev. Lett. 85, 1910 (2000).

${ }^{9}$ K. A. Fichthorn and M. Scheffler, Phys. Rev. Lett. 84, 5371 (2000).

${ }^{10}$ N. Knorr, H. Brune, M. Epple, A. Hirstein, M. A. Schneider, and K. Kern, Phys. Rev. B 65, 115420 (2002).

${ }^{11}$ V. S. Stepanyuk, L. Niebergall, R. C. Longo, W. Hergert, and P. Bruno, Phys. Rev. B 70, 075414 (2004).

${ }^{12}$ V. S. Stepanyuk, A. N. Baranov, D. V. Tsivlin, W. Hergert, P. Bruno, N. Knorr, M. A. Schneider, and K. Kern, Phys. Rev. B 68, 205410 (2003).
${ }^{13}$ F. Silly, M. Pivetta, M. Ternes, F. Patthey, J. P. Pelz, and W.-D. Schneider, Phys. Rev. Lett. 92, 016101 (2004).

${ }^{14}$ F. Silly, M. Pivetta, M. Ternes, F. Patthey, J. P. Pelz, and W.-D. Schneider, New J. Phys. 6, 16 (2004).

${ }^{15}$ K. A. Fichthorn and W. H. Weinberg, J. Chem. Phys. 95, 1090 (1991).

${ }^{16}$ K. A. Fichthorn, M. L. Merrick, and M. Scheffler, Phys. Rev. B 68, 041404(R) (2003).

${ }^{17}$ P. Hyldgaard and M. Persson, J. Phys.: Condens. Matter 12, L13 (2000).

${ }^{18}$ T. L. Einstein and J. R. Schrieffer, Phys. Rev. B 7, 3629 (1973).

${ }^{19}$ B. Gumhalter and W. Brenig, Surf. Sci. 336, 326 (1995).

${ }^{20}$ K. Wildberger, V. S. Stepanyuk, P. Lang, R. Zeller, and P. H. Dederichs, Phys. Rev. Lett. 75, 509 (1995).

${ }^{21}$ P. Lang, L. Nordstrom, K. Wildberger, R. Zeller, P. H. Dederichs, and T. Hoshino, Phys. Rev. B 53, 9092 (1996); D. G. Pettifor, Commun. Phys. 15, 193 (1985).

${ }^{22}$ V. S. Stepanyuk, L. Niebergall, W. Hergert, and P. Bruno, Phys. Rev. Lett. 94, 187201 (2005).

${ }^{23}$ V. S. Stepanyuk, A. N. Baranov, W. Hergert, and P. Bruno, Phys. Rev. B 68, 205422 (2003).

${ }^{24}$ N. N. Negulyaev, V. S. Stepanyuk, W. Hergert, H. Fangohr, and P. Bruno, Surf. Sci. 600, L58 (2006). 